\title{
DNA Methylation of Gene Expression in Acanthamoeba castellanii Encystation
}

\author{
Eun-Kyung Moon', Yeonchul Hong², Hae-Ahm Lee ${ }^{3}$, Fu-Shi Quan', Hyun-Hee Kong 4,* \\ ${ }^{1}$ Department of Medical Zoology, Kyung Hee University School of Medicine, Seoul 02447, Korea; ${ }^{2}$ Department of Parasitology and Tropical \\ Medicine, Kyungpook National University School of Medicine, Daegu 41944, Korea; ${ }^{3}$ Department of Pharmacology, Kyungpook National University \\ School of Medicine, Daegu 41944, Korea; ${ }^{2}$ Department of Parasitology, Dong-A University College of Medicine, Busan 49201, Korea
}

\begin{abstract}
Encystation mediating cyst specific cysteine proteinase (CSCP) of Acanthamoeba castellanii is expressed remarkably during encystation. However, the molecular mechanism involved in the regulation of CSCP gene expression remains unclear. In this study, we focused on epigenetic regulation of gene expression during encystation of Acanthamoeba. To evaluate methylation as a potential mechanism involved in the regulation of CSCP expression, we first investigated the correlation between promoter methylation status of CSCP gene and its expression. A 2,878 bp of promoter sequence of CSCP gene was amplified by PCR. Three CpG islands (island 1-3) were detected in this sequence using bioinformatics tools. Methylation of $\mathrm{CpG}$ island in trophozoites and cysts was measured by bisulfite sequence PCR. CSCP promoter methylation of $\mathrm{CpG}$ island $1(1,633 \mathrm{bp})$ was found in $8.2 \%$ of trophozoites and $7.3 \%$ of cysts. Methylation of CpG island 2 (625 bp) was observed in $4.2 \%$ of trophozoites and 5.8\% of cysts. Methylation of CpG island 3 (367 bp) in trophozoites and cysts was both $3.6 \%$. These results suggest that DNA methylation system is present in CSCP gene expression of Acanthamoeba. In addition, the expression of encystation mediating CSCP is correlated with promoter CpG island 1 hypomethylation.
\end{abstract}

Key words: Acanthamoeba castellanii, encystation, epigenetic regulation

\section{INTRODUCTION}

Acanthamoeba cysts are resistant to physical, chemical, radiological conditions, and chemotherapeutic agents [1,2]. Acanthamoeba infection can be difficult to treat due to the cyst stage. In order to understand the encystation mechanism and improve treatment for Acanthamoeba infection, several encystation-mediating factors have been studied. Comparative microarray analysis has shown that 701 genes are up-regulated during the encystation of Acanthamoeba [3]. Autophagosome related proteins, cyst wall related genes, and cyst specific serine and cysteine proteinases have been reported to be needed for the formation of cyst of Acanthamoeba [4-8]. However, regulation mechanisms of these encystation mediating genes are currently unknown.

Gene regulation has a wide range of mechanisms, including

\footnotetext{
- Received 21 February 2017, revised 13 March 2017, accepted 3 April 2017.

*Corresponding author (hhkong@dau.ac.kr)

(c) 2017, Korean Society for Parasitology and Tropical Medicine

This is an Open Access article distributed under the terms of the Creative Commons Attribution Non-Commercial License (http://creativecommons.org/licenses/by-nc/4.0) which permits unrestricted non-commercial use, distribution, and reproduction in any medium, provided the original work is properly cited.
}

increasing or decreasing the production of specific gene products. Gene expression can be modulated by transcriptional initiation, RNA processing, and post-translation of protein. In higher eukaryotes, epigenetic regulation responsible for gene expression has been widely studied. Epigenetics is associated with microRNA expression, DNA-protein interaction, suppression of transposable element mobility, cellular differentiation, embryogenesis, X-chromosome inactivation, and genomic imprinting [9]. Epigenetic modifications can be grouped into 3 main categories: DNA methylation, histone modification, and nucleosome positioning [9]. DNA methylation regulates a number of important biological functions such as chromatin structure, silencing of gene expression, parental imprinting, and chromosome X inactivation [10-12]. Knowledge of DNA methylation and its effects on gene expression in parasites are poorly understood. In protozoan parasite Entamoeba histolytica, 5-methyl cytosine and DNA methyltransferase 2 have been identified [13]. In Plasmodium falciparum, methylated cytosine and a single functional DNA methyltransferase have been reported [14]. Geyer et al. [15] have shown evidence of cytosine methylation in Schistosoma mansoni, suggesting the presence of a functional DNA methylation machinery. However, methylat- 
ed cytosine and DNA methyltransferase have not been reported in Acanthamoeba yet, although protein arginine methyltransferase 5 has been identified [16].

In this study, we found that 5-azacytidine, an inhibitor of DNA methyltransferase, could reduce the encystation ratio of Acanthamoeba, suggesting that DNA methylation system might be associated with the encystation process in Acanthamoeba. To determine the effect of DNA methylation on gene expression, promoter methylation status and gene expression of cyst specific cysteine proteinase of Acanthamoeba (GenBank accession no. JQ253375) were examined in this study.

\section{MATERIALS AND METHODS}

\section{Amoeba cultivation and encystation}

Acanthamoeba castellanii Castellani was obtained from the American Type Culture Collection (ATCC 30011). Trophozoites of A. castellanii were axenically cultured in PYG medium ( $2 \%$ proteose peptone, $0.1 \%$ yeast extract, and $100 \mathrm{mM}$ glucose) at $25^{\circ} \mathrm{C}$ in an incubator. Encystation of A. castellanii was induced in an encystation media $\left(0.1 \mathrm{M} \mathrm{KCl}, 0.008 \mathrm{M} \mathrm{MgSO}_{4}\right.$, $0.0004 \mathrm{M} \mathrm{CaCl}_{2}$, and $0.02 \mathrm{M}$ 2-amino-2-methyl-1,3-propanediol ( $\mathrm{pH}$ 9.00)) for 3 days.

\section{5-azacytidine demethylation treatment}

Acanthamoeba trophozoites were treated with $10 \mu \mathrm{M}$ of 5-azacytidine (Sigma-Aldrich, St. Louis, Missouri, USA) and incubated in PYG media or encystment media for 3 days. Mature cysts were counted under a light microscope after treating them with $0.5 \%$ SDS. Encystation ratios were calculated. Data are expressed as means $\pm S D$ of the means from 3 independent experiments. Statistical significance was analyzed using unpaired Student's $t$-test. A $P$-value of less than 0.05 was considered statistically significant.

\section{DNA extraction and promoter prediction}

Genomic DNA of Acanthamoeba trophozoite was purified using QIAamp genomic DNA kits (Qiagen, Hilden, Germany) according to the manufacturer's instructions. Promoter sequences were obtained using GenomeWalker kit (BD Biosciences Clontech, Palo Alto, California, USA), following the protocol recommended by the manufacturer. Briefly, the first step was the construction of pools of adaptor-ligated genomic DNA fragments using 1 of 4 restriction enzymes (EcoRV, DraI, PvuII, or SspI). The first PCR amplification was performed with outer
Table 1. Primers used in this study

\begin{tabular}{|c|c|c|}
\hline Primer name & Sequence $\left(5^{\prime} \rightarrow 3\right)$ & $\begin{array}{l}\text { Product } \\
\text { size (bp) }\end{array}$ \\
\hline CP-GSP 1 & GAAGAAGAAGACGATGTTGATCCTCAT & 2,904 \\
\hline CP-GSP 2 & CGTGTTGTTCTIITGTAGTITGTGAGT & 2,877 \\
\hline Promoter-F1 & $\begin{array}{l}\text { F: TTGGTATTATAATATATAATATTATTA } \\
\text { R: AAATTACTCACTACACACTAACAACAA }\end{array}$ & 322 \\
\hline Promoter-F2 & 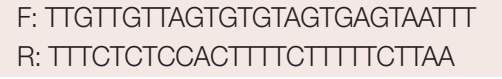 & 502 \\
\hline Promoter-F3 & $\begin{array}{l}\text { F: TTAAGAAAAAGAAAAGTGGAGAGAA } \\
\text { R: TAAACCTTAATATTATAATAACTAAA }\end{array}$ & 539 \\
\hline Promoter-F4 & $\begin{array}{l}\text { F: TTTAGTTATTATAATATTAAGGTTA } \\
\text { R: TTATAAATATAACTCTTCAACTAACCT }\end{array}$ & 320 \\
\hline Promoter-F5 & $\begin{array}{l}\text { F: AGGTTAGTTGAAGAGTTATATTTATAA } \\
\text { R: AACAAAAAAACCCACAAATACCAATAA }\end{array}$ & 455 \\
\hline Promoter-F6 & $\begin{array}{l}\text { F: TTATTGGTATTIGTGGGTIIIITGTT } \\
\text { R: TAATAATTAACTTAAAACTAAATATAA }\end{array}$ & 421 \\
\hline Promoter-F7 & $\begin{array}{l}\text { F: TTATATTAGTITAAGTTAATTATTA } \\
\text { R: TATTATTCTIITATAATTATAAATT }\end{array}$ & 415 \\
\hline
\end{tabular}

adaptor primer (AP1) provided in the kit and cysteine proteinasegene-specific primer (CP-GSP 1) (Table 1). Nested GSP (CPGSP 2) (Table 1) and nested adaptor primer (AP2) were used in sequential PCR to amplify the promoter. For primary PCR, the following cycling parameters were used: 7 cycles of $25 \mathrm{sec}$ at $94^{\circ} \mathrm{C}$ and $4 \mathrm{~min}$ at $72^{\circ} \mathrm{C}$ followed by 32 cycles of $25 \mathrm{sec}$ at $94^{\circ} \mathrm{C}$ and $4 \mathrm{~min}$ at $67^{\circ} \mathrm{C}$ with a final extension at $67^{\circ} \mathrm{C}$ for an additional $4 \mathrm{~min}$. Primary PCR products were diluted 1:50 and $1 \mu \mathrm{l}$ of the diluted primary PCR product was used as template for secondary amplification. Cycling parameters for the secondary PCR were the same as those used for the primary PCR except that the first and second steps consisted of 5 and $22 \mathrm{cy}$ cles, respectively. A single band of 3,000 bp was obtained after PCR amplification. It was cloned into pGEM-T easy vector system (Promega, Madison, Wisconsin, USA). Two clones were picked for plasmid DNA purification for sequencing analysis.

\section{$\mathrm{CpG}$ islands and bisulfite sequencing PCR}

Promoter CpG islands and bisulfite sequencing PCR primers were designed based on Methprimer site (Table 1). The primers were designed that they didn't contain any CpGs in order to facilitate their binding to both methylated and unmethylated sequences. Genomic DNA of Acanthamoeba trophozoite and cyst were prepared with QIAamp genomic DNA kits (Qiagen), and Bisulfite kit (Qiagen) was used for bisulfite sequencing PCR. Bisulfite conversion and subsequent purification were performed according to the respective protocols. Seven PCR products (F1-7) were cloned into the PGEM-T easy 
vector system, and each ten clones were picked for sequencing analysis.

\section{RESULTS}

\section{Effect of 5-azacytidine on Acanthamoeba}

In order to identify the effect of 5-azacytidine on growth and encystation of Acanthamoeba, trophozoites were cultured with $10 \mu \mathrm{M}$ 5-azacytidine for 3 days. Results showed that 5-azacytidine had limited effect on the growth of Acanthamoeba compared to untreated control (Fig. 1A, B, E). However, when trophozoites were transferred to encystment media, 5-azacytidine reduced the encystation ratio (Fig. 1C, D, F). Several trophozoites were observed (arrows) after induction into encystment (Fig. 1D). The number of mature cysts was significantly reduced in 5-azacytidine treated cells (52.2\%) compared to that in control cells (80.5\%) (Fig. 1F). These data indicated that, although 5-azacytidine did not significantly affect Acanthamoeba growth, it reduced encystation ratio.

\section{Cloning of cysteine proteinase promoter and prediction of $\mathrm{CpG}$ islands}

To identify the promoter region of cyst specific cysteine proteinase (CSCP) of Acanthamoeba, a 2,878 bp segment at the 5 -flaking region of CSCP was amplified by PCR using specific primers (Table 1; Fig. 2). CG dinucleotides in the promoter region were underlined. Scanning for $\mathrm{CpG}$ islands in the submitted sequence revealed $3 \mathrm{CpG}$ islands in Methprimer (Fig. $3 \mathrm{~A})$. The $3 \mathrm{CpG}$ island regions were at least $200 \mathrm{bp}$ with a GC percentage greater than $50 \%$. The observed-to-expected $\mathrm{CpG}$ ratio was greater than $60 \%$. The $3 \mathrm{CpG}$ islands were divided into 7 fragments for bisulfite sequencing PCR (Fig. 3B). A total of 14 bisulfite sequencing PCR primers were then designed according to CpG islands (Table 1).

\section{Identification of DNA methylation by bisulfite treatment and sequencing}

To determine the effect of promoter methylation on gene expression, we performed bisulfite sequencing for encystation mediating CSCP in Acanthamoeba trophozoite and cyst. In trophozoite, $8.2 \%, 4.2 \%$, and 3.6\% methylation were observed in CpG island 1, CpG island 2, and CpG island 3, respectively. At cyst stage, 7.3\%, 5.8\%, and 3.6\% methylation were observed in CpG island 1, CpG island 2, and CpG island 3, respectively. Total promoter methylation status of CSCP in trophozoite was
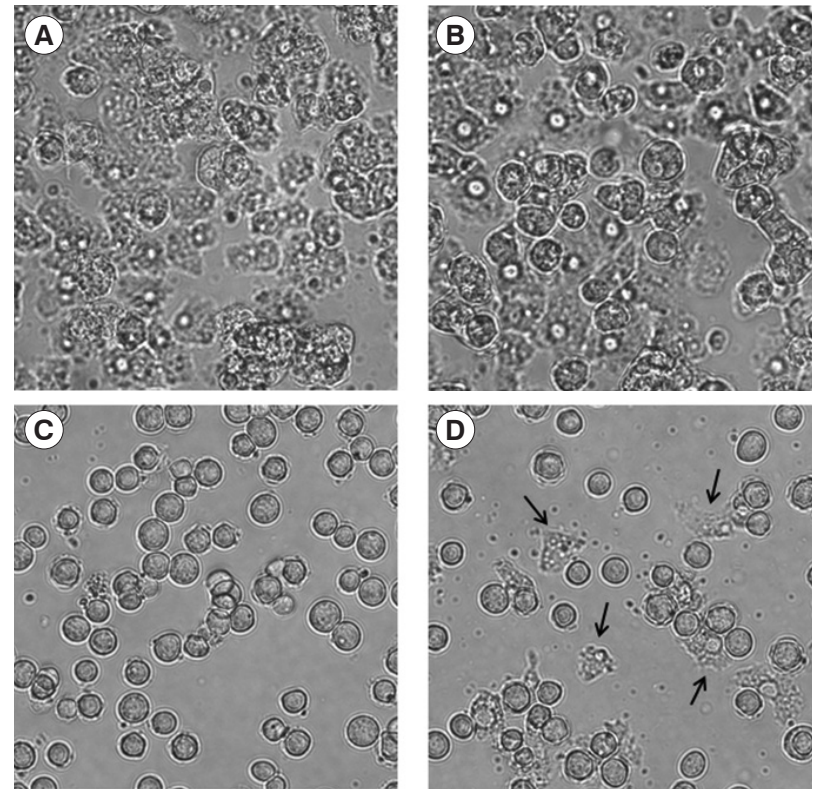

$\mathrm{E}$

\section{$\mathrm{F}$}
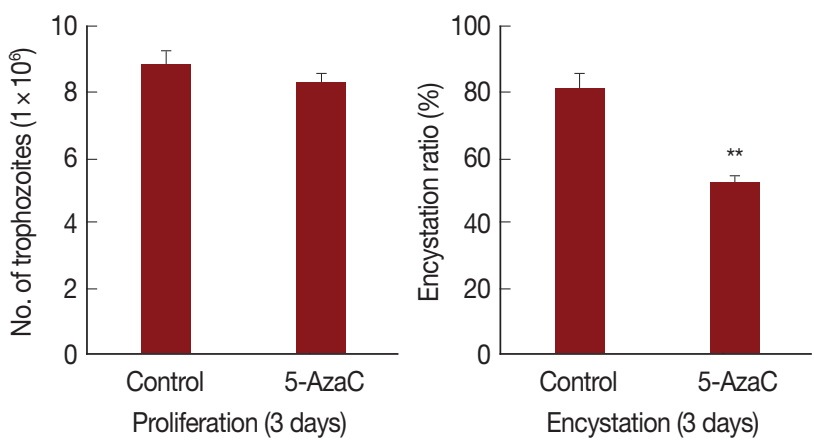

Fig. 1. Effect of 5-azacytidine on proliferation and encystation. Acanthamoeba trophozoites were cultured in PYG media without 5-azycytidine (A) or with 5-azacytidine (B) for 3 days. Trophozoites were transferred to encystment media and encystation was induced for 3 days without 5 -azycytidine (C) or with 5-azacytidine (D). The 10 $\mu \mathrm{M} 5$-azacytidine has limited effect on the growth of Acanthamoeba (E). However, encystation ratio was reduced in 5-azacytidine treated cells (52.2\%) compared to that in control cells (80.5\%) (F). Arrows indicate remained trophozoites after the induction of encystation (D). Values indicate means $( \pm S D)$ from 3 experiments.

approximately equal to that in cyst. However, $\mathrm{CpG}$ island 1 of trophozoite showed higher methylation (8.2\%) than that of cyst (7.3\%) (Fig. 4). The length of CpG island 1 was 1,633 bp. There were 148 CpG sites.

\section{DISCUSSION}

Cyst specific cysteine proteinase (CSCP) of Acanthamoeba is highly expressed during encystation. The expression of this 
GG_GGCCGCGGGAATTCGATTACTATAGGGCACGCGTGGTCGACGGCCCGGGCTGGTATCACAACACACAACACCATCAA GTGCGCGAGTGTGT GCGTACTTCATGGCCTCCGTGCGGCGGTGGTGGGTCCACATCACGCCGTCCATCAGCTGAACGTTGT TCTCCTGCGAGCATTACAGGAACACCACCCAACCACAGCCAATCATGCCCGGGGTTAATCGTGACTTGCCGCACAGTTCGC TGCACCAGGTGTGTCGGTGGCTCGTGCCTTGCAGGCGGCGATCATCCTCTTGACTT GCGCCACATCGCAACCCAGCGGCTTT TCGCACCT GATGTGTTGAGCACACCGCCAACGGTTGTTGTTAGTGTGCAGTGAGCAATTCTTACAACCATTTAATACCTCCG CAGCCCGCAATT GCGGGCGGGTGACGG_GAGAGTGGTGTGGGGCCTCGACGTACAGCACGTGCTTCTTGTGCTGGG_GG GGCGATGGTGAGGGACTCGTGGAGGTGGGTCGGCGTGGGGATGTAGACGGCGTCAACGTCGGGGTCACGCAACAGCTCC TCGTAGCTGCCGTAGGCCTT GGCGATCCCCT GCTCCTTGCCCCATTCT GTCGCGCGGCACCACCAAATCAAAGTCAATCGGG TGAGTCTCCGGAGCT ACAAGATGGAAAGATCAGCCTACCCTGGGCGCGTTCGAGGGAGCGGCTGGCGACGGCAACGATG GACGACCCCT CGGCCTTCTTGACCGTCTGGACGAACACTTTGGCGATTTGGGCAGTACCCAACACGCCCCACCTCACTTCTT GCGCCATCTITTGGGGTTTGCTTCGTTTAAGAAAAAGAAAAGTGGAGAGAAAAAAAAATTCACGTACGGCACGTGCGCCG AGCCCACCGCCCGCAACAGCGCACAGGACCCCAAAACATGAAGCACCGCGCGGACGAACGGGGAAGTGTACGAATTTCCT GCTCGGACTACTCCGGCAGCCGAACGAACGAATAGCGCACTTTCTTTTGGTTTCGCCACAGAGCCTGGCCCAATTGGGCAC ACGAAGAGGCAACGTCTCGCCGGCACGCAGCCTGCCAGCGTTACCTACCCAAGAACGAAAGCGAAGAGCACAAGAGCGA AAGCGAAAGCGAAGCGCGACAGAACGAAACCGATGGCCACAACAACCATGCCCACACAGCGGGTGGTGCAGCTATGGAC GCCCGACCCGGCTCTGAACGCCACCAACGTGCGTCGACGACCCCTCGCCCCGTGCTCCCACCTCCTCGAGCT GCAGAGGCG ATCAGCT GAAGAGGACCACGGCTCCACCGTCACTCGGTTTCGCAAACCGGTTCCTCCGCCCAGCCACCACAACACCAAGGC CCACGTCGAAGTGGCGTCCCTGCTCGACATCT GCGCCCAGGCCATACTCCTCCACCAACACCGCCAGCGCACCACCAGAAA ATCGTCGCCGACAGCACATCATAATGCGCCATCATCGGGTCGTCATCATCACTGGGCCCAGCAGAAGTAGGAGGCCTCCCT CTCGGTCGTGGCGACGACGGGTCAATTTGATATGGATCT GCT GCCGCGGGAACTGCGCGCGTACCTGAGCGAGCACTGGC TGTGCGTTGTGTGCCAGCGCCCAACACGAGGCCAGCT GAAGAGCCACACCCACAATTCCCACCATGACAAGGGCCGACCG GGTGCCAACTACCCCGGCT GGT GT CCTGCCT GCGTCCAGCGT GCGTCCAGCTT GCCCCGCGGTCACTGTGGTGCTTGGGTG ATGTGGGT GGGCCGAACTAAAGCAAAT GCTTGTGTGGTGGTGGTGTTCCGCT GAACAGGGCTGTGCT ATTGCCAGGCCGA CGGATGTCAGGCGTGT AACTCTACGTGGCTGTGCTTGACCTGCGGCAAGGTCTGCT GCAGCACTTCTTCTCACGGTACGATC GAATTCTCATTITTTCTCGTTATAGTCATCATCGCTGGCGCTTACATT GCTTCTCTGATGATCATCATCAGAGCATT CATT CGA CCACT CGATGATGGAATCGCACATGCTTGCTTGGAAAGGTACAAACCGCGTCACTGGCACTTGTGGGTTCCTCTGTCTGAC GCTCTCCTCCACCCGATCGGGTGGCCCATGTCTGTACAGTCGGGCCAAGACCCTTTCAGCAAGCCGCAGCTTACGACGCAG GCGACCCCGGT ATCT GGT GCTT CGCCT GCT CGCGGT ACGTCG GCGAGGCCAAGCT GCACCTCCACCTTCAGACGCT GGGCC TCACGGAGCCCTCCGCT GCCGCCGCCGCTGACTGCGATGTCAGCGAGAGATAAGTGTTTCAAAAACAAACCTCACTTTTTT GATTTAGTTGTGGTTCATGTTGTTCTATCTCTGTTTCCAACAGAATTCGTGGGTTCTGCAGCAAGGGTT GATGTGGTGTCAG TTCGAAACAGCAGTTGACTCCGAAGCCAATGAGGTTTCTTIACATTTAGCTCCAAGCCAATCACCACGCACTGGACATAGCC GGTCCGCGGAACGCACCGCGGTAAGGAGTGACGGAGGT GAACT GGCCAGTGGCGGCCGCT CCG GCCCTCCCTGGCGAAA GCGAACGCCCACGAGGAGAAACTCTCAGAGGCT GACCGTGTCCGGGGAACTCCTCGCCCGAGTCCGGATCTCCTCAT GAC ATCGACCTGACGTGCCTCCAGCCCGCTGGGTATAAAATGGACCGGGCCAGGCCCCTAAGCTCTTCATCTT CATCCTCCATGT AAAGCAAAGCGAAGCCACACTTTCGCTACTTCTTCTACTTCTTCTTCTTCTTCCCTTCGTTCAGGCTT CGATCCAT CGAT CGA AGACAACGAAAACACACAAAAAACTCACAAACTACAAAAAGAACAACA CGATG .

Fig. 2. Promoter region of CSCP. Nucleotide sequence of CSCP promoter region was amplified by PCR using GenomeWalker Kit. This DNA is 2,878 bp in length at the 5'-flaking region of CSCP for bisulfite sequencing PCR. CG dinucleotides in promoter region were underlined.

A

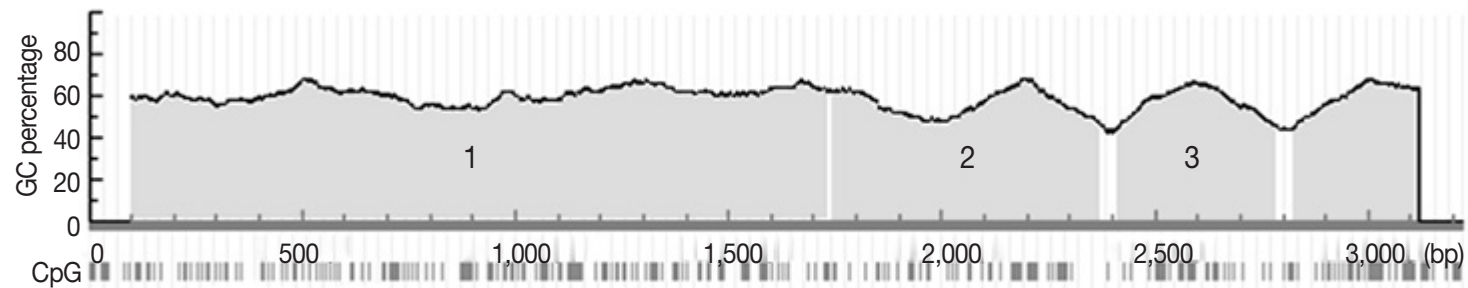

B

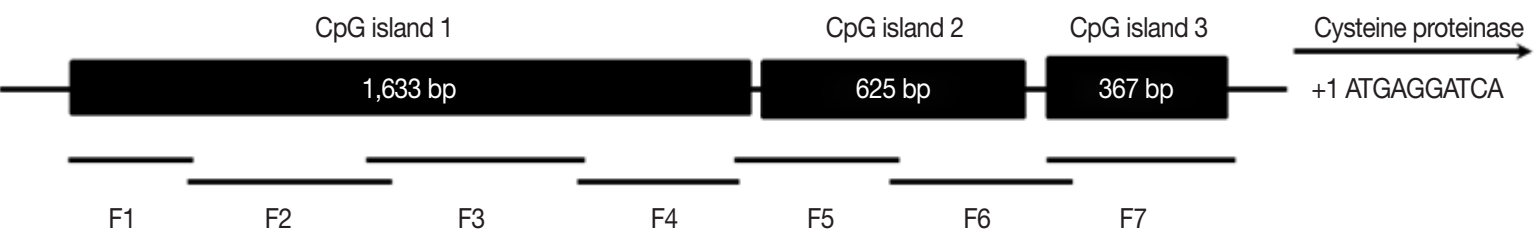

Fig. 3. Bioinformatic analysis of CSCP promoter region. Three CpG islands (island 1, 1,633 bp; island 2, 625 bp; island 3, 367 bp) of CSCP promoter region were detected by bioinformatics analysis (A). The promoter region was divided into 7 fragments $(B)$. Primers for bisulfite sequencing PCR were designed based on Methprimer.

gene is necessary for encystation of Acanthamoeba [3,8]. In order to understand the regulation of CSCP expression during encystation, epigenetic regulation was examined by analyzing the correlation between promoter methylation status and gene expression in Acanthamoeba.

As an inhibitor of DNA methyltransferase, 5-azacytidine in- 


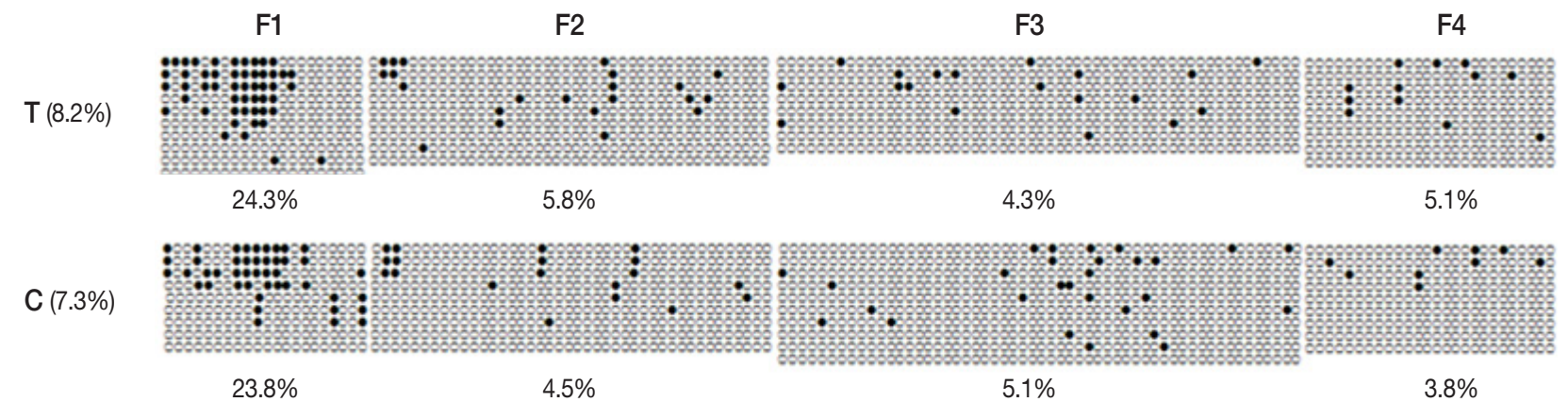

Fig. 4. Methylation status of CpG island 1 of CSCP promoter. Methylation of promoter CpG island 1 of CSCP in trophozoite was compared to that in cyst by bisulfite sequencing PCR. In trophozoite CpG island 1, 8.2\% methylation was observed while 7.3\% methylation was observed in cyst. CpG island 1 of trophozoite showed higher methylation than that of cyst. T, trophozoite; C, cyst; $\bullet$, methylated CpG site; and o, unmethylated CpG site.

hibited the formation of mature cyst in Acanthamoeba (Fig. 1). It has been reported that $E$. histolytica heat shock protein 100 can be induced by 5-azacytidine [17]. In S. mansoni, it has been reported that 5-azacytidine can disrupt egg production and egg maturation [15]. These results suggest that DNA methylation might play essential roles in gene regulation in parasites. Until now, the role of DNA methylation in gene expression of Acanthamoeba has not been reported yet. Therefore, we screened the correlation between DNA methylation and gene regulation in encysting Acanthamoeba.

Here, we showed cytosine methylation in the promoter of CSCP of Acanthamoeba (Fig. 4). CpG island 1 of CSCP promoter in trophozoite showed higher methylation than that in cyst. $\mathrm{CpG}$ islands are regions with high frequency of $\mathrm{CpG}$ (or $\mathrm{CG}$ ) sites. $\mathrm{CpG}$ island is usually defined as a region of at least 200 bp with GC percentage greater than 50\%, and with an observed-to-expected $\mathrm{CPG}$ ratio that is greater than $60 \%$. DNA methylation, occurring in the context of $\mathrm{CpG}$ dinucleotide, has profound effect on gene expression by modifying the accessibility of DNA to transcription factors [18].

Although we do not know why CpG island 1 is more effective than other CpG islands, activation of CSCP of Acanthamoeba correlated with $\mathrm{CpG}$ island 1 hypomethylation might play a role. Total promoter methylation status of CSCP in trophozoite is approximately equal to that of cyst. Other factors might also affect gene expression of CSCP in encysting Acanthamoeba. However, CpG island 1 was composed of 1,633 bp including 148 CpG sites. CpG island 2 was composed of 625 bp including $45 \mathrm{CpG}$ sites while $\mathrm{CpG}$ island 3 was composed of $367 \mathrm{bp}$ including $25 \mathrm{CpG}$ sites (data not shown). Further studies are needed to determine the transcriptional regulation by differen- tial promoter methylation and examine the relationship between promoter methylation status of selected CpG islands in trophozoite and cyst [19].

The detection of methylated cytosine in this study suggests the presence of a DNA methylation system for gene regulation in encysting Acanthamoeba. This also provides an opportunity to investigate the effect of DNA methylation on gene expression associated with encystation. Further studies on epigenetic gene regulation in Acanthamoeba will provide important information to understand gene expression for encystation and amoebiasis control.

\section{ACKNOWLEDGMENT}

This research was supported by the Basic Science Research Program of the National Research Foundation of Korea (NRF) funded by the Ministry of Education (grant no. 2014R1A1A 2058405).

\section{CONFLICT OF INTEREST}

We have no conflict of interest related with this study.

\section{REFERENCES}

1. Aksozek A, McClellan K, Howard K, Niederkorn JY, Alizadeh H. Resistance of Acanthamoeba castellanii cysts to physical, chemical, and radiological conditions. J Parasitol 2002; 88: 621-623.

2. Turner NA, Russell AD, Furr JR, Lloyd D. Emergence of resistance to biocides during differentiation of Acanthamoeba castellanii. J Antimicrob Chemother 2000; 46: 27-34.

3. Moon EK, Xuan YH, Chung DI, Hong Y, Kong HH. Microarray 
analysis of differentially expressed genes between cysts and trophozoites of Acanthamoeba castellanii. Korean J Parasitol 2011; 49: 341-347.

4. Moon EK, Chung DI, Hong YC, Kong HH. Autophagy protein 8 mediating autophagosome in encysting Acanthamoeba. Mol Biochem Parasitol 2009; 168: 43-48.

5. Song SM, Han BI, Moon EK, Lee YR, Yu HS, Jha BK, Danne DB, Kong HH, Chung DI, Hong Y. Autophagy protein 16-mediated autophagy is required for the encystation of Acanthamoeba castellanii. Mol Biochem Parasitol 2012; 183: 158-165.

6. Moon EK, Hong Y, Chung DI, Goo YK, Kong HH. Down-regulation of cellulose synthase inhibits the formation of endocysts in Acanthamoeba. Korean J Parasitol 2014; 52: 131-135.

7. Moon EK, Chung DI, Hong YC, Kong HH. Characterization of a serine proteinase mediating encystation of Acanthamoeba. Eukaryot Cell 2008; 7: 1513-1517.

8. Moon EK, Hong Y, Chung DI, Kong HH. Cysteine protease involving in autophagosomal degradation of mitochondria during encystation of Acanthamoeba. Mol Biochem Parasitol 2012; 185: 121-126.

9. Portela A, Esteller M. Epigenetic modifications and human disease. Nat Biotechnol 2010; 28: 1057-1068.

10. Robertson KD. DNA methylation and chromatin - unraveling the tangled web. Oncogene 2002; 21: 5361-5379.

11. Attwood JT, Yung RL, Richardson BC. DNA methylation and the regulation of gene transcription. Cell Mol Life Sci 2002; 59: 241257.

12. Goto T, Monk M. Regulation of X-chromosome inactivation in development in mice and humans. Microbiol Mol Biol Rev 1998; 62: 362-378.

13. Fisher O, Siman-Tov R, Ankri S. Characterization of cytosine methylated regions and 5-cytosine DNA methyltransferase (Ehmeth) in the protozoan parasite Entamoeba histolytica. Nucleic Acids Res 2004; 32: 287-297.

14. Ponts N, Fu L, Harris EY, Zhang J, Chung DW, Cervantes MC, Prudhomme J, Atanasova-Penichon V, Zehraoui E, Bunnik EM, Rodrigues EM, Lonardi S, Hicks GR, Wang Y, Le Roch KG. Genome-wide mapping of DNA methylation in the human malaria parasite Plasmodium falciparum. Cell Host Microbe 2013; 14: 696-706.

15. Geyer KK, Rodríguez López CM, Chalmers IW, Munshi SE, Truscott M, Heald J, Wilkinson MJ, Hoffmann KF. Cytosine methylation regulates oviposition in the pathogenic blood fluke Schistosoma mansoni. Nat Commun 2011; 2: 424.

16. Moon EK, Hong Y, Chung DI, Goo YK, Kong HH. Identification of protein arginine methyltransferase 5 as a regulator for encystation of Acanthamoeba. Korean J Parasitol 2016; 54: 133-138.

17. Bernes S, Siman-Tov R, Ankri S. Epigenetic and classical activation of Entamoeba histolytica heat shock protein 100 (EHsp100) expression. FEBS Lett 2005; 579: 6395-6402.

18. Jones PA, Liang G. Rethinking how DNA methylation patterns are maintained. Nat Rev Genet 2009; 10: 805-811.

19. Lim JH, Kim SP, Gabrielson E, Park YB, Park JW, Kwon TK. Activation of human cancer/testis antigen gene, XAGE-1, in tumor cells is correlated with CpG island hypomethylation. Int J Cancer 2005; 116: 200-206. 